\title{
The study of reducing soil mechanical deformation in tilling
}

\author{
GaliaKokieva ${ }^{1, *}$, Martha Ivanova $^{2}$, and Varvara Trofimova $^{1}$ \\ ${ }^{1}$ The Arctic State Agrotechnological University, 3, sh. Sergelyakhskoe $3 \mathrm{~km}, 677007$, Yakutsk, the \\ Republic of Sakha (Yakutia), Russia \\ ${ }^{2}$ The North-Eastern Federal University named after M.K. Ammosov, 58, st.Belinskogo, 677007, \\ Yakutsk, the Republic of Sakha (Yakutia), Russia
}

\begin{abstract}
Due to the use of heavy machines, mechanical deformation with the inevitable deterioration in its physico-biological properties is undergoing in agriculture of heavy machines and subfall layers of the soil. The density of the arable and subframe horizons increases, decreases, due to the moisture in the soil. The productivity of cultural plants is influenced by effective fertility not only arable layer, but also subfectant, so it is necessary to take into account the effects on them of agricultural machinery and tractors. At the first stage, the soil particles in close proximity to the paw are shifted forward, forcing the lying layers in front to shrink, when the voltages in the soil reaches the limit values, at some distance from the sock of the paw blades, its shift occurs. At the second stage, the soil begins to intensively shifted forward and move along the surface of the worker. In this regard, the soil resistance to the movement of individual parts of the working body will be different. At the moment when there is an intensive compression of the soil and its shift, the most loaded elements will be the cutting edge and the nose part of the paw, the least - wings of the paws and racks.
\end{abstract}

\section{Introduction}

One of the possible measures to prevent the compaction of the subsurface layers is technological advancement of tilling the top soil. Model studies have showed that in creating and maintaining the optimal texture density and content in the tilled layer of the studied chernozem (taking into account the requirements of vertical development of agronomic variables), it was possible, to an extent, to optimize the water collection capacity and soil discharge, soil and the atmosphere air exchange, hasten the emerging crops and improve the phenological parameters of plants, yields by at least $20 \%$ compared to the control. The use the damping properties of loose soil is envisaged in order to reduce or prevent the spread of mechanical deformation along the soil crossover to depth. The driving speed of the implements has been the same $-6.3 \ldots 6.5 \mathrm{~km} / \mathrm{h}$. The observations over the change of soil density to a depth of $60 \mathrm{~cm}$ have revealed different deformation of its layers, depending on the intensity of breaking up and the pressure of tillage outfits (see

\footnotetext{
${ }^{*}$ Corresponding author: kokievagalia@mail.ru
} 
table). The density of the upper layers after first plowing becomes less than before tilling. The density of subsurface layers increases under the machine-tractor aggregates mass. The weight of the MTF-50 tractor is about 3 tons, the DT-75M tractor - about 7 tons; the pressure on the soil of the former exceeds $0.1 \mathrm{mPa}$, the latter is almost by one half less.

\section{Results and discussion}

There are various formulas $[1,4,7]$ for testing soil resistance. We have calculated the total shareresistance having tested its components, depending on the loading of the share elements (lip, sharewings, tine).

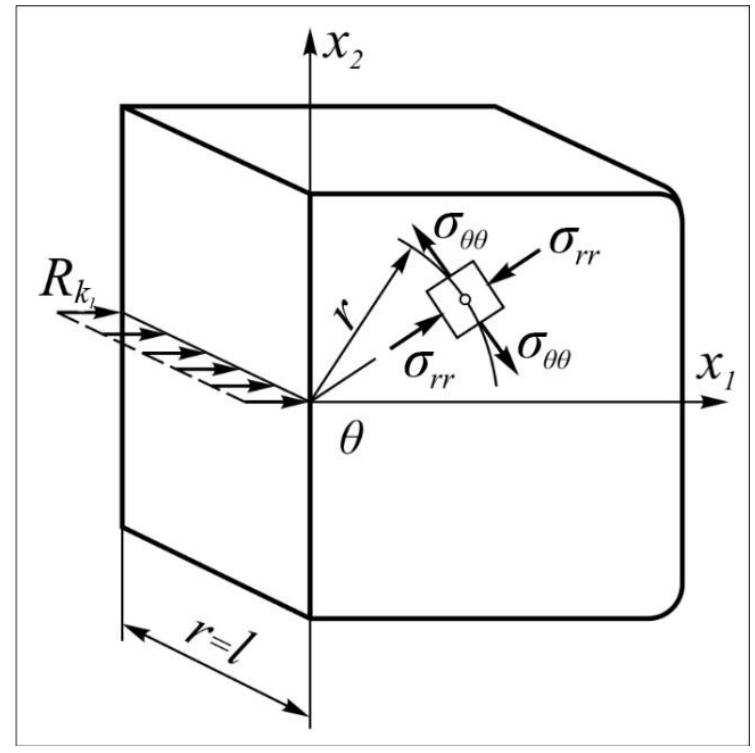

Fig. 1. Soil loading diagram.

Considering that the soil has internal bonds between single particles, it can be a deformable viscoelastic body with predominantly elasticity. Therefore, we have applied the theory of elasticity with a sufficient degree of accuracy. To calculate the soil resistance to the movement of the cutting edge of the cultivator share, the plane elasticity in rotational positions has been solved. The normal pressure on the edges is determined in the same way as for a flat wedge $[2,5,15]$. Therefore, we consider the deformation of the soil by one wing of the share. The resistance of the total cutting edge of the entire share has been found as the sum of the projections of the resistance forces of the wings to the direction of movement. The layer of soil, cultivated by the edge of the wing of the share, can be represented with sufficient accuracy as a semi-infinite slab (Fig. 1). Then the stresses arising in the soil.

$$
\begin{gathered}
\sigma_{r r}=\frac{1}{r} \frac{\partial F}{\partial r}+\frac{1}{r^{2}} \cdot \frac{\partial^{2} F}{\partial \theta^{2}} ; \\
\sigma_{\theta \theta}=\frac{\partial^{2} F}{d r^{2}} \\
\sigma_{r \theta}=-\frac{\partial}{\partial r}\left(\frac{1}{r} \cdot \frac{\partial F}{\partial \theta}\right),
\end{gathered}
$$


where $\sigma_{r r}, \sigma_{\theta \theta}, \sigma_{r \theta}$ - the main stresses, acting along the radial line and shearing; $\mathrm{F}(r, \theta)$ is the Airy stress function in rotational positions $[8,9]$. The change in soil pressure along the length of the wedge tip should be noted $[3,10]$. Therefore, for the cutting edge of the share (the lip of the share and the adjoining part of the wing for the sharpening width), we assume, with some approximation, a linear distribution of the load acting on the straight edge of the semi-infinite slab, and the mass loads equal to zero due to the relatively small depth of tilling. Then the stress distribution in the soil layer is will be radial, and the Airy function is symmetric relating to the measured vectorial angle $\theta$ :

$$
F(r, \theta)=\mathrm{A} r \sin \theta,
$$

where $A$ is a constant; $r$ - the distance to the point at which the stress is determined.

According to the accepted assumptions $\sigma_{\theta \theta}=0$ and $\sigma_{\mathrm{r}} \theta=0$, and the simultaneous solution of (1) and (2) gives

$$
\sigma_{r r}=\frac{r A}{r} \cos \theta .
$$

In determining the constant $A$ in equation (3), we consider the equilibrium condition of the soil layer between the external $d R_{k_{1}}$ and internal forces, which we findby means of the stress $\sigma_{r r}$, and the sectional area of the elementary volume of the semi-infinite slab (or soil layer) $d A_{1}=r d \theta t$ ( $t$ - the thickness of semi-infinite slab), $d R h_{1}=\sigma_{r r} \cos \theta d A_{1} . \sigma \mathrm{rr}=\mathrm{rA} /$ $\mathrm{r} \cos : \theta$.

Considering that the share mainly forces on the soil lying above the edge, we consider the stress $\sigma_{r r}$ in the layers bounded by the $\mathrm{X}_{1}$ and $\mathrm{X}_{2}$ axes, the limits of integration are from 0 to $\pi / 2$.

Then

$$
R_{k_{1}}=\int_{0}^{\pi / 2} \sigma_{r r} \cos \theta r t d \theta
$$

from equations (3) and (4) we find

$$
A=2 R_{k_{1}} /(\pi t) \text {. }
$$

Substituting the values of $A$ into equation (3), we obtain the soil resistance to the movement of the share edge at the normal to the lip:

$$
R_{k_{1}}=r t \pi /(4 \cos \theta) \text {. }
$$

Soil resistance to movement of the entire edge

$$
R_{k_{1}}=\frac{\sigma_{r r} r t \pi}{2 \cos \theta} \cos \gamma,
$$

where $\gamma$ is half of the share nose angle.

The stresses are infinitely large at the application of the force $R_{k_{1}}$. Therefore, ground flow is inevitable close to it. However, at some distance from the place of application of force $[9,12]$, according to the principle of locality, formula (6) can be suitable for practical calculations. Moreover, the shear of the soil layer occurs at a distance $l^{\prime}$ from the share lip at $\theta=0$ [6]. Therefore, it is advisable to refer the limiting values to this area and consider the resistance $R_{k}$ depending on the stress $\sigma_{r r}$ arising in at maximum stresses at the shear moment, i.e.

$$
\sigma_{r r}=\sigma_{c o m p},
$$

where $\sigma_{\text {comp }}$ - is the compressive stress for a specific type of soil and working conditions. 
To determine the resistance of the soil to the movement of the share wings $-R_{s h}$ and the tine $-R_{t}$, a well-known technique has been used $[8,10,13]$.

The soil resistance force to the movement of the share wings

$$
\begin{aligned}
R_{s h} & =\left\{\frac{a l \rho v_{s} v_{s h} \sin \beta}{\sin (\beta+\psi)}-\left[\cos \psi \sin (\beta+\psi) \operatorname{tg} \varphi_{1} \cos \beta\right]+\operatorname{alb} \beta g \cos ^{2} \beta \operatorname{tg} \varphi_{1}\right\} \sin \gamma \\
& +\sigma_{\text {comp }} B b \sin \beta+a^{2} B \rho g \operatorname{tg} \varphi_{2} \operatorname{ctg} \psi
\end{aligned}
$$

wherel is the length of the share lip; $\rho$ is the soil density; $\varphi_{1}-$ angle of external friction of the soil to share; $b$ is the width of the share wing; $g$ is gravity factor; $\varphi_{2}-$ angle of internal friction of the soil.

\begin{tabular}{|c|c|c|c|c|c|c|c|}
\hline \multirow{2}{*}{ Tilling sequence } & \multicolumn{7}{|c|}{ Soil layer, cm } \\
\hline & $0 \ldots 10$ & $10 \ldots 20$ & $20 \ldots 30$ & $30 \ldots 40$ & $40 \ldots 50$ & $50 \ldots 60$ & $30 \ldots 60$ \\
\hline Before tilling & 1.02 & 1.32 & 1.27 & 1.42 & 1.44 & 1.52 & 1.46 \\
\hline \multicolumn{8}{|c|}{$\begin{array}{l}\text { After first plowing } \\
\text { byanaggregatewithtract } \\
\text { or: }\end{array}$} \\
\hline \multirow{2}{*}{ MTF-50 } & 1.02 & 1.30 & 1.29 & 1.42 & 1.44 & 1.52 & 1.46 \\
\hline & $\overline{1.07}$ & $\overline{1.24}$ & $\overline{1.27}$ & $\overline{1.42}$ & $\overline{1.44}$ & $\overline{1.54}$ & $\overline{1.47}$ \\
\hline \multirow{2}{*}{ DT-75M } & 1.00 & 1.24 & 1.23 & 1.43 & 1.55 & 1.60 & 1.52 \\
\hline & $\overline{1.04}$ & $\overline{1.19}$ & $\overline{1.30}$ & $\overline{1.44}$ & $\overline{1.54}$ & $\overline{1.66}$ & $\overline{1.54}$ \\
\hline \multicolumn{8}{|c|}{$\begin{array}{l}\text { After tilling the first } \\
\text { plowed field by the } \\
\text { aggregate withtractor } \\
\text { DT-75M: }\end{array}$} \\
\hline \multirow{2}{*}{ MTF-50 } & 1.16 & 1.21 & 1.32 & 1.44 & 1.48 & 1.56 & 1.49 \\
\hline & $\overline{1.15}$ & $\overline{1.18}$ & $\overline{1.28}$ & $\overline{1.43}$ & $\overline{1.47}$ & $\overline{1.54}$ & $\overline{1.48}$ \\
\hline \multirow{2}{*}{ DT-75M } & 1.17 & 1.22 & 1.43 & 1.50 & 1.61 & 1.69 & 1.60 \\
\hline & $\overline{1.16}$ & $\overline{1.18}$ & $\overline{1.30}$ & $\overline{1.44}$ & $\overline{1.54}$ & $\overline{1.66}$ & $\overline{1.54}$ \\
\hline $\begin{array}{l}\text { After tilling the } \\
\text { unplowed field }\end{array}$ & 1.15 & 1.21 & 1.33 & \multicolumn{2}{|c|}{1.49} & 1.54 & 1.50 \\
\hline
\end{tabular}

Table 1. Soil density, $\mathrm{g} / \mathrm{cm} 3$, depending on the methods of its tilling. 


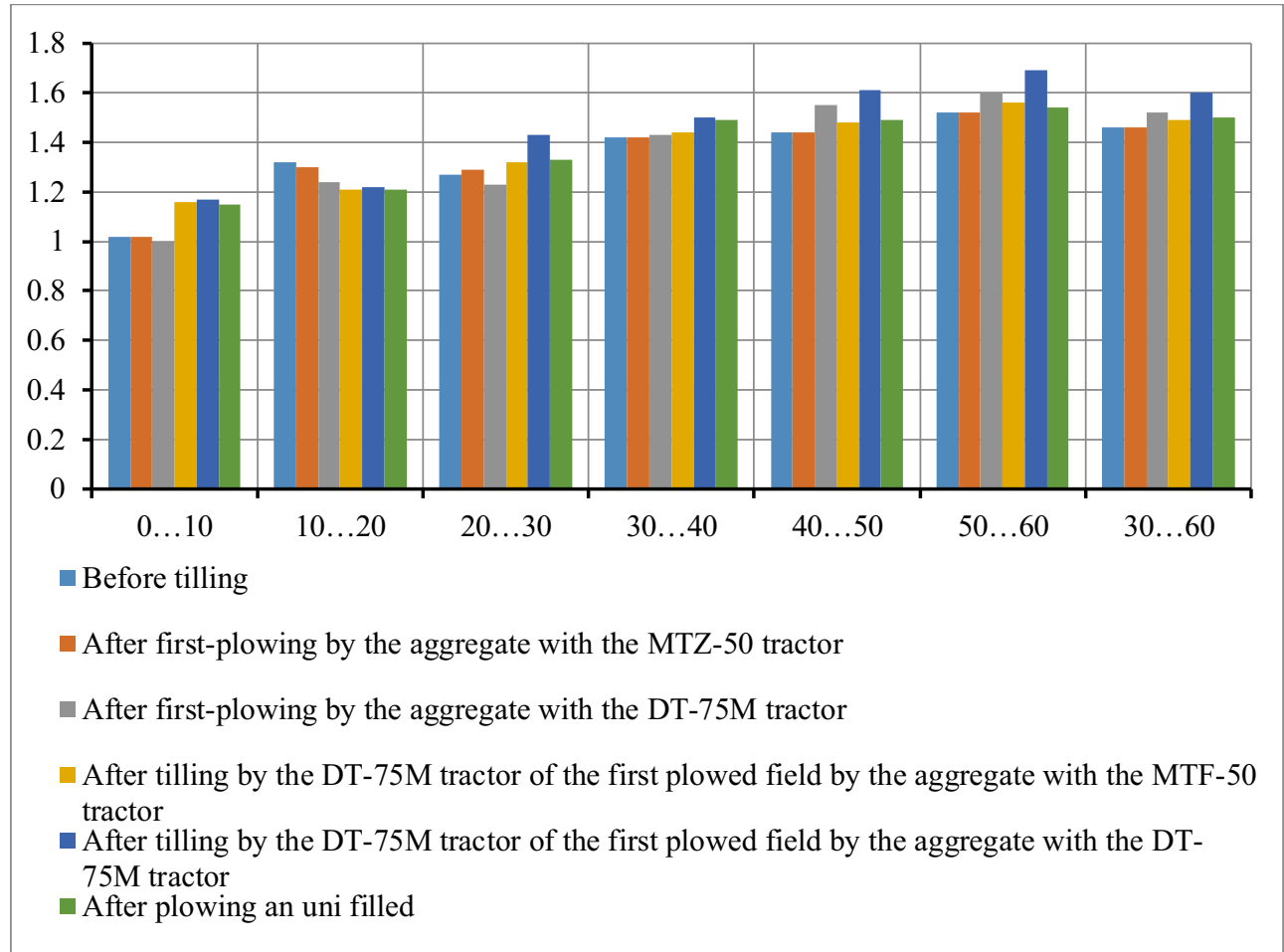

The analysis of the subsurface soil layers density at a depth of $30 \ldots 60 \mathrm{~cm}$ (where its change is more clearly observed in tilling the top layer under the influence of machinetractor aggregates) shows that when the deeply first-plowed fields $(14 \ldots 16 \mathrm{~cm})$ tilled by a large tractor (drawbar category 3 ), the subsurface layers are compacted less than in tilling first-plowed one $(5 \ldots 6 \mathrm{~cm})$ with the same tractor. A lighter tractor (drawbar category 1.4) firms the soil less in first plowing than a large one. The highest yield $(30.6 \mathrm{t} / \mathrm{ha})$ of herbage (oats, peas, sunflower) has been obtained in a field tilled by a DT-75M tractor, after first plowing to a depth of $14 \ldots 16 \mathrm{~cm}$ by a MTF-50 tractor aggregate $[6,11,14]$.

The proposed method of tilling the topsoil as one of the measures to prevent the subsoil prerconsolidation ensures the improvement or preservation in them the favorable physical conditions for plants, avoiding the extent of mechanical deformations to depth due to the damping property of loose soil against the ground, drive system pressureoftractors and agricultural machines.

Table 2. Productivity, $\mathrm{t} / \mathrm{ha}$, herbage of non-perennial crops' mixture at a depth of first plowing $(5 \ldots$ 6) / (14 ... 16) cm and tilling (20 cm).

\begin{tabular}{|l|c|}
\hline $\begin{array}{c}\text { Tilling by DT-75M after first plowing by the aggregatewith } \\
\text { a tractor }\end{array}$ & \\
\hline MTF-50 & $28.20 / 30.60$ \\
\hline DT-75M & $26.96 / 28.11$ \\
\hline The same in unplowed soil & 24.96 \\
\hline Lsd 0.95 & 5.40 \\
\hline
\end{tabular}


On the basis of the foregoing, it is possible to formulate the following essential agrotechnical requirements for engineering and facilities used in the cultivating of spiked cereals on chernozem soils of medium and heavy texture to:

- improve the structure of the top and especially the seed layers. The sizes of the aggregates in the top seed layer should be adjusted to $0.25 \ldots 20 \mathrm{~mm}$ [1] predominantly aggregates $5 \ldots 20 \mathrm{~mm}$ in the top and $0.25 \ldots 5 \mathrm{~mm}$ - in the seed layers. The aggregates larger than $20 \mathrm{~mm}$ and smaller than $0.25 \mathrm{~mm}$ [1] from the top layer are not used; dust content (particles less than $0.25 \mathrm{~mm}$ ) in the seed layer should not exceed $15 \%$;

- improve the bulk density of the top layer (should be $1.0 \ldots 1.3 \mathrm{~g} / \mathrm{cm} 3$ ); to form a moderately compressed $(1.2 \ldots 1.3 \mathrm{~g} / \mathrm{cm} 3)$ over-seed layer (to "block" the unproductive moisture flow) and less loose seed and sub-seed layers $(1.1 \ldots 1.2 \mathrm{~g} / \mathrm{cm} 3)$. The density of the top and subsurface layers should not be higher than 1.3 and lower than $1.0 \mathrm{~g} / \mathrm{cm} 3$.

Crumbs of medium and large sizes (larger than $3 \mathrm{~mm}$ ) in chernozems are formed not by the action of subsurface mechanisms - sequential physical, mechanical and chemical sealing, but as a result of crushing under the action of roots, volumetric changes, mainly mechanical tilling.

The latter applies equally to the bulk density of the soil, the size of changing of which is very wide depending on the used tools and the tilling methods: the studied chernozem - 0.9 ... $1.5 \mathrm{~g} / \mathrm{cm} 3$ (low level - straight after plowing and harrowing, high - after repeated rolling by the tractor wheels of drawbar category 3-5).

These proposals can be realized by improving tilling technologies and all new machines establishment. The possibilities of the first direction are limited, because multiple runs of different implements will be required, which is undesirable. Moreover, by the use of the existing tools, it is practically difficult to break up the soil thoroughly in the seed layers and to reduce simultaneously the bulk density in the sub-seed layers. For these reasons, we give preference to the second direction.

\section{Conclusion}

In agricultural production, tilling is the most energy-intensive technology, including a number of technological operations. Tilling is carried out with tine cultivators designed to break up of soil and weed destruction. In poor tilling, up to half of the sown seeds lose their germinability. The cultivators with blunt shares have an increased draught. The technical state of the cultivator shares determines both the unevenness of the tilling depth and the weed infestation of soil. That is why the problem of increasing the resource of the working bodies of tillage machines is very urgent. A well-conditioned chernozem soil can practically be adjusted to the required ratio of structural components and bulk density, using our own resources, without additional facilities (soil stabilizer), using only looseningfirming working bodies of various designs and taking into account the tilling conditions. To optimize the structural composition and bulk density, it is most effective to use a combined machine that tills the soil, sows seeds and applies fertilizer in one run. The analysis of the known combined machines' work has showed that none of them can cope with this task. For its implementation, most probably, it is necessary to use other technological processes or select the luckiest combination of unpowered and powered tillage tools. One of the possible types of machines for making an optimal top soil layer is a combined one, consisting of two parts. The first is a working tool that ensures the optimal structure of the rooting (sub-seed) soil layer. It is not easy to obtain such a structure, because the density of chernozems before sowing, as a rule, only slightly exceeds the required one. Therefore, tilling should only slightly reduce the density of its lower layers. The root layer cannot be intensively tilled, as this can lead to its compaction during the rooting, which is unacceptable. 


\section{References}

1. I.B. Borisenko, M.V. Meznikova, Bulletin of Agrarian Science of the Don, 4(52), 19-28 (2020)

2. O.G..Chertov, M.A. Nadporozhskaya, Computer Research and Modeling, 8(2), 391-399 (2016)

3. S.E. Fedorov, Tractors and agricultural machines, 2, 78-82 (2018)

4. M.B. Khalilov, Scientific life, 4, 57-68 (2018)

5. A.A. Konishchev, E.N. Konischeva, Agrarian Bulletin of the Upper Volga Region, 3 (24), 18-24 (2018)

6. V.A. Korolev, A.I. Gromovik, O.K. Borontov, Eurasian Soil Science, 1, 95-101 (2016)

7. I.A. Lontseva, N.V. Soboleva, AgroEkoInfo, 37(3), 38 (2019)

8. T. Machado, K. Lanyas, 36, 646-655 (2016) [CrossRef] [Google Scholar]

9. G.A. Okunev, N.A. Kuznetsov, APK of Russia, 75(1), 89-95 (2016)

10. N.M. Ozhegov, D.B. Slinko, D.A. Kaposhko, Repair. Recovery. Modernization, 11, 43-48 (2018) doi: 10.31044/1684-2561-2018-0-11-43-48

11. D. Teravest, P.R. Wandschneider, C. Thierfelder, etc. Agricultural Systems, 171, 23-35 (2019)

12. A.R. Valiev, Bulletin of Kazan State Agrarian University, 12-3 (45), 54-60 (2017) doi: 10.12737/article_5ald9587a0d852.23012684.

13. E.M. Yudina, A.S. Brusentsov, collected papers of Int. scientific-practical conf. (February 25, 2016, Tomsk). In 3 parts, part 3 - Ufa: AETERNA, 101-104 (2016)

14. E.M. Yudina, The XX Int. scientific-production conf. proceedings (Belgorod, May 23-25, 2016), vol. 2-Belgorod: Publishing House of FSBEI HE Belgorod SAU, 141-142 (2016)

15. D.I. Zolotarevskaya, International Journal of Applied and Fundamental Research, 7-5, 754-763 (2016) 